\title{
Optically Induced Indirect Photonic Transitions in a Slow Light Photonic Crystal Waveguide
}

\author{
Michel Castellanos Muñoz, ${ }^{1, *}$ Alexander Yu. Petrov, ${ }^{1}$ Liam O’Faolain, ${ }^{2}$ Juntao Li, ${ }^{3,2, \dagger}$ \\ Thomas F. Krauss, ${ }^{4,2}$ and Manfred Eich ${ }^{1}$ \\ ${ }^{1}$ Institute of Optical and Electronic Materials, Hamburg University of Technology, Hamburg 21073, Germany \\ ${ }^{2}$ SUPA, School of Physics and Astronomy, University of St. Andrews, St. Andrews, Fife KY16 9SS, United Kingdom \\ ${ }^{3}$ State Key Laboratory of Optoelectronic Materials \& Technology, Sun Yat-sen University, Guangzhou 510275, China \\ ${ }^{4}$ Department of Physics, University of York, Heslington, York, YO10 5DD, United Kingdom
}

(Received 9 August 2013; published 6 February 2014)

\begin{abstract}
We demonstrate indirect photonic transitions in a silicon slow light photonic crystal waveguide. The transitions are driven by an optically generated refractive index front that moves along the waveguide and interacts with a signal pulse copropagating in the structure. We experimentally confirm a theoretical model which indicates that the ratio of the frequency and wave vector shifts associated with the indirect photonic transition is identical to the propagation velocity of the refractive index front. The physical origin of the transitions achieved here is fundamentally different than in previously proposed refractive index modulation concepts with fixed temporal and spatial modulation frequencies; as here, the interaction with the refractive index front results in a Doppler-like signal frequency and wave vector shift. Consequently, the bandwidth over which perfect mode frequency and wave vector matching is achieved is not intrinsically limited by the shape of the photonic bands, and tuning of the indirect photonic transitions is possible without any need for geometrical modifications of the structure. Our device is genuinely nonreciprocal, as it provides different frequency shifts for co- and counterpropagating signal and index fronts.
\end{abstract}

The process of inducing a frequency and a wave vector shift on an optical signal, such that it undergoes a transition between two modes of a photonic structure, has been referred to as an indirect photonic transition in the recent literature [1-5]. Indirect photonic transitions are not only of fundamental physical interest, but may constitute the operating principle for the realization of integrated nonreciprocal optical isolators without using magneto-optical effects [4]. An indirect photonic transition between two modes $\left(\omega_{0}, k_{0}\right)$ and $\left(\omega_{1}, k_{1}\right)$ can be achieved by modulating the refractive index of the structure both temporally with a frequency $\Omega=\omega_{1}-\omega_{0}$, and spatially with a spatial frequency $q=k_{1}-k_{0}[1-4,6]$, which can be explained as a three wave mixing process in a nonlinear system [6]. However, while the modulation can be designed to match the mode separation $(\Omega, q)$ for a particular optical frequency $\omega_{0}$ in principle, the matching no longer exists for neighboring frequencies $\omega_{0}+\Delta \omega$ if the bands involved in the transition are not completely parallel throughout the desired frequency bandwidth [2-4]. There exists, thus, an intrinsic limitation on the bandwidth over which a transition is possible, imposed by the group velocity mismatch of the bands. There are other practical limits: the frequency shift $\Omega$ is limited by the achievable modulation frequencies to a maximum of tens of $\mathrm{GHz}$, and the typically required spatial modulation frequency $q$ must be encoded in the geometry of the device, since it cannot be matched by the spatial frequency of a traveling $\mathrm{GHz}$ electric signal [4].
Thus, tuning of the indirect photonic transition is only possible by varying the geometry of the device.

In this Letter, we achieve indirect photonic transitions that are driven by an optically generated refractive index front traveling along a slow light photonic crystal waveguide. This front provides the required temporal and spatial index change. The physical origin of the transitions achieved here is fundamentally different from any previously mentioned refractive index modulation concept with fixed temporal and spatial modulation frequencies. As we will show below, our concept overcomes the aforementioned intrinsic bandwidth limitation imposed by the shape of the photonic bands, which is a key feature that opens up the possibility of inducing transitions between fast and slow bands. Furthermore, the magnitude of the induced shifts in frequency and wave vector, and hence the magnitude of the indirect photonic transition, can be tuned by varying the propagation velocity of the refractive index front. There is no need for encoding a spatial modulation frequency in the geometry of the device, and tuning is possible without any geometrical variation. Here, we show shifts of $\Omega=$ $120 \mathrm{GHz}$ and $q=0.05 \mu \mathrm{m}^{-1}$ achieved with a refractive index change of $\Delta n=-3 \times 10^{-3}$ in a $396 \mu \mathrm{m}$ long structure, and also suggest that a slight modification of the experimental setup (not of the device itself) may allow us to achieve shifts of $\Omega=700 \mathrm{GHz}$ and $q=0.5 \mu \mathrm{m}^{-1}$ in the same device by applying the same $\Delta n$. 
The indirect photonic transitions shown here rely on the interaction of an optical signal with a moving free-carrier plasma front in a semiconductor [7-10], which leads to a moving refractive index front via the carrier-plasma dispersion effect [11]. In this case, the front velocity may exceed the speed of light in vacuum, since no material is actually moving. For example, it was previously shown that light confined to a photonic structure can be shifted in frequency if a refractive index change $\Delta n$ is applied to the entire structure while the light is still confined within it [9,12-16]. This case corresponds to a refractive index front traveling at infinite velocity, and can be classified as a direct photonic transition, since $\Delta n$ causes a change in frequency $\omega$ (proportional to $-\Delta n$ ) but leaves the wave vector $k$ of the light signal unaltered. In contrast, we achieve here an indirect photonic transition, where both $\omega$ and $k$ are changed in a single step, by creating a refractive index front of finite velocity. Remarkably, the induced frequency shift can be tuned independently of $\Delta n$, it can be larger in magnitude and even have an opposite sign compared to the shift achieved for an infinite front velocity (provided $\Delta n$ is the same in both cases).

Indirect photonic transitions in a photonic crystal waveguide have now also been demonstrated in a two-step process [5], and offer exciting opportunities for ultrafast delay applications [5,17]. Here, we study indirect photonic transitions that occur in a single step and investigate the parameter space required for tuning their magnitude. We generate a refractive index front that propagates along a slow light photonic crystal waveguide and measure the frequency shift it induces on a copropagating signal pulse upon transmission through the moving front. We experimentally study several combinations of the propagation velocities of the refractive index front and the signal, and find agreement with the theoretical prediction, indicating that the shifts of signal wavelength and wave vector induced upon interaction with the moving front are determined by both the propagation velocity of the front and the dispersion curve of the system. Therefore, by exploiting the flexibility in dispersion design provided by slow light waveguides [18,19], a variety of indirect transitions that create a novel potential for light control can be achieved, as we discuss below.

Figure 1 shows a schematic representation of the experiment. A high power switching pulse propagating through a silicon photonic crystal waveguide generates free carriers by two-photon absorption, and induces a corresponding change of the refractive index $\Delta n_{\mathrm{FC}}$ due to the carrier plasma dispersion effect [11]. Accordingly, a refractive index front moves at the group velocity of the switching pulse and interacts with a signal copropagating in the waveguide. Here, we choose the difference between the group velocities of the front and the signal to be large compared to the pulse duration and the propagation length, such that either the refractive index front completely overtakes the signal, as shown in Fig. 1(a), or that the

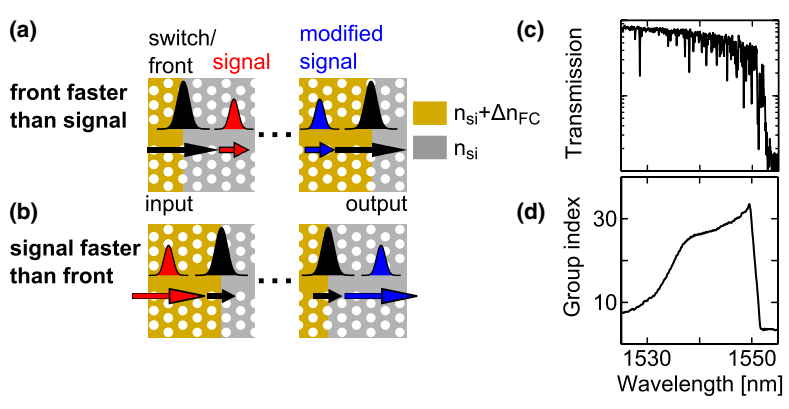

Fig. 1 (color online). (a)-(b) Schematic of the experiment. A switching pulse with high peak power generates free carriers in the silicon by two-photon absorption and, consequently, induces a change of refractive index which propagates with the velocity of the switch. (a) The switching pulse is faster than the signal, corresponding to Fig. 2(a). (b) The signal pulse is faster than the switch, corresponding to Figs. 2(c) and 2(e). (c) Transmission and (d) group index measured on the fabricated photonic crystal waveguide.

signal overtakes the front, as shown in Fig 1(b). It can be seen from Fig. 1 that the index change experienced by the signal upon interaction with the moving refractive index front is $\Delta n=\Delta n_{\mathrm{FC}}$ for Fig. 1(a) and $\Delta n=-\Delta n_{\mathrm{FC}}$ for Fig. 1(b). Recalling that the frequency shift is proportional to $-\Delta n$ for a direct transition [9,12-16], one could expect a blue and a red frequency shift for the cases of Figs. 1(a) and 1(b), respectively. However, we induce here an indirect transition, and a more careful analysis is necessary in order to correctly describe it.

In the rest frame of the moving refractive index front, the front can be chosen to lie at the position $z_{f}^{\prime}=0$. If, in this frame, $\omega_{1}^{\prime}, k_{1}^{\prime}, \omega_{2}^{\prime}, k_{2}^{\prime}$ denote the frequencies and wave vectors of the signal on each side of the front, the product of the four vectors is

$$
\begin{aligned}
\left(\frac{\omega_{2}^{\prime}-\omega_{1}^{\prime}}{c}, k_{2}^{\prime}-k_{1}^{\prime}\right)\left(c t^{\prime}, z_{f}^{\prime}\right)= & \left(\omega_{2}^{\prime}-\omega_{1}^{\prime}\right) t^{\prime} \\
& -\left(k_{2}^{\prime}-k_{1}^{\prime}\right) z_{f}^{\prime}=0,
\end{aligned}
$$

where $c$ is the light velocity in vacuum and $t^{\prime}$ is the time coordinate in the rest frame of the front. This product is invariant under Lorentz transformations. Thus, the time and space dependent phase of the signal is continuous at the position of the moving front, independently of the reference frame of the observer. If we now denote the frequencies and wave vectors in the laboratory frame by $\omega_{1}, k_{1}, \omega_{2}, k_{2}$, the velocity of the refractive index front in the laboratory frame by $v_{f}$ and its position by $z_{f}$, we find

$$
\begin{aligned}
\left(\omega_{2}-\omega_{1}\right) t-\left(k_{2}-k_{1}\right) z_{f} & =0, \\
\frac{\omega_{2}-\omega_{1}}{k_{2}-k_{1}}=\frac{z_{f}}{t}=\frac{t v_{f}}{t} & =v_{f} .
\end{aligned}
$$

Equation (2) indicates that, as observed in the laboratory frame, the ratio of the changes of frequency and wave 
vector induced by the interaction with the moving front is identical to the velocity at which the front propagates.

We first apply this result to the case where a refractive index front moving at velocity $v_{f}$ completely overtakes a signal pulse, as shown in Fig. 1(a), and use a graphical representation [20,21], shown in Fig. 2(a), in order to determine the magnitude of the indirect photonic transition induced by the front. The solid and dashed curves in Fig. 2(a) schematically show the dispersion bands of a silicon photonic crystal waveguide for silicon refractive indices $n_{\mathrm{si}}$ and $n_{\mathrm{si}}+\Delta n_{\mathrm{FC}}$, respectively. Initially, the signal pulse travels in a waveguide characterized by $n_{\mathrm{si}}$, and its initial state $\left(\omega_{1}, k_{1}\right)$ lies on the corresponding dispersion curve. Next, we draw all points $(\omega, k)$ which fulfill the phase continuity condition from Eq. (2) by plotting a phase continuity line with a slope equal to the traveling velocity of the front $v_{f}$. After being overtaken by the refractive index front, the signal pulse travels in a waveguide characterized by $n_{\mathrm{si}}+\Delta n_{\mathrm{FC}}$. Its final state $\left(\omega_{2}, k_{2}\right)$ is determined graphically from the crossing point of the phase continuity line and the dashed dispersion curve. Next, we analyze the inverse configuration, corresponding to Fig. 1(b), where the signal pulse overtakes the refractive

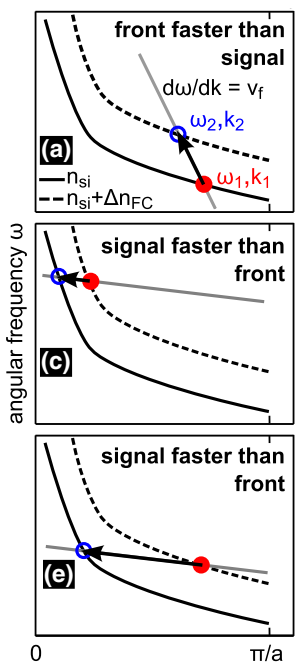

Wavevector $\mathrm{k}$

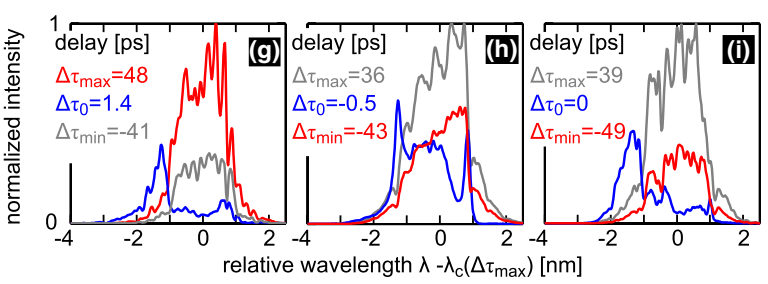

Fig. 2 (color online). Schematic representation of transitions, measured relative center wavelength, and selected signal spectra in the different configurations: (a),(b),(g) $n_{g \text {,signal }}=29$, $n_{g, \text { switch }}=11$, correspond to Fig. 1(a); (c),(d),(h) $n_{g \text {,signal }}=11$, $n_{g \text {,switch }}=32$, correspond to Fig. 1(b); (e),(f),(i) $n_{g \text {,signal }}=26$, $n_{g \text {,switch }}=32$, correspond to Fig. 1(b). index front. In contrast to the previous case, we show in Fig. 2(c) that the initial state of the signal now lies on the band corresponding to $n_{\mathrm{si}}+\Delta n_{\mathrm{FC}}$, while the final state lies on the band corresponding to $n_{\mathrm{si}}$. Remarkably, the induced indirect transition again leads to a blueshift of the signal frequency, in contrast to the redshift that one would expect for a direct photonic transition, as we discussed above. The observation of a blueshift in this configuration therefore unequivocally proves the achievement of an indirect photonic transition. Finally, we study a third configuration, shown in Fig. 2(e), where, again, the signal pulse overtakes the refractive index front. The velocity and refractive index contrast of the front are kept the same as in Fig. 2(c); however, we now choose a different initial state of the signal with lower signal propagation velocity. A larger frequency blueshift is expected in this case, which is confirmed by comparing Figs. 2(c) and 2(e).

We note that, in contrast to [1-4], the phase continuity line does not unambiguously determine one, but rather a whole set of possible final states $(\omega, k)$. Out of this set, such transitions will take place for which the final state corresponds to a photonic mode of the system. As long as this second condition is satisfied, there are no intrinsic limitations on the bandwidth over which a perfect mode frequency and wave vector matching can be achieved. Furthermore, the velocity of the refractive index front, which is identical to the velocity of the switching pulse, can be tuned via the operating point of the slow light waveguide. Thus, the indirect photonic transitions are tunable without the need for any geometrical modifications. The efficiency of the indirect transitions is determined by the spatial overlap of the modes involved and by the adiabaticity of the refractive index change; a smooth index front will adiabatically transfer energy to the mode shifted by the free carriers, and here, the transition to that mode has the highest efficiency. We also note that a vertical phase continuity line in Fig. 2(a), which describes a direct transition, would lead to a frequency change smaller than that actually achieved for the indirect transition shown.

We fabricated a single line defect photonic crystal waveguide consisting of a hexagonal lattice of air holes in silicon [22,23]. The measured transmission and group index [24] are shown in Figs. 1(c) and 1(d), respectively.

We implemented the configuration of Fig. 2(a) by choosing group indices of $n_{g, \text { switch }}=11$ and $n_{g \text {,signal }}=29$. The waveguide, with a length of $396 \mu \mathrm{m}$, introduces a relative pulse delay of $\Delta \tau_{\mathrm{WG}}=-22 \mathrm{ps}$, where we define $\Delta \tau<0$ if the switching pulse runs ahead of the signal. The peak power of the signal pulse is low enough to avoid nonlinear effects, and the pulse lengths are measured to be $t_{\text {signal }} \leq 7 \mathrm{ps}$ and $t_{\text {switch }}=2$ ps.

For a peak power of the switching pulse in the waveguide $P_{\text {switch }}=11.20 \mathrm{~W}$, we record the signal spectrum $S(\lambda, \Delta \tau)$ at the output of the waveguide for several values of the relative pulse delay $\Delta \tau$ at the input. For each measurement, 
we calculate the center wavelength of the signal spectrum $\lambda_{c}(\Delta \tau)$ :

$$
\lambda_{c}(\Delta \tau)=\frac{\int \lambda \times S(\lambda, \Delta \tau) d \lambda}{\int S(\lambda, \Delta \tau) d \lambda}
$$

We plot in Fig. 2(b) the relative center wavelength $\Delta \lambda_{c}(\Delta \tau)=\lambda_{c}(\Delta \tau)-\lambda_{c}\left(\Delta \tau_{\max }\right)$, where $\Delta \tau_{\max }$ is the largest recorded relative pulse delay. We choose $\lambda_{c}\left(\Delta \tau_{\max }\right)$ as a reference because at $\Delta \tau_{\max }$ the signal does not interact with the moving front, and it propagates through a waveguide with unperturbed silicon refractive index.

In the yellow and green regions of Fig. 2(b) the fast switch cannot overtake the slow signal, in the yellow region due to the large initial pulse separation, in the green one because the switch runs ahead. The signal does not interact with the moving front in these regions, and, therefore, no wavelength shift $\left(\Delta \lambda_{c} \approx 0\right)$ is observed. In Fig. $2(\mathrm{~g})$ we plot the signal spectra, relative to $\lambda_{c}\left(\Delta \tau_{\max }\right)$, measured at $\Delta \tau_{\max }=48$ ps and $\Delta \tau_{\min }=-41 \mathrm{ps}$. At $\Delta \tau_{\max }$ the signal propagates through a waveguide with unperturbed $n_{\mathrm{si}}$. At $\Delta \tau_{\min }$, however, the signal interacts with the carriers generated by the switch, resulting in additional losses [11] and a lower peak transmission.

In the gray region of Fig. 2(b), the moving index front overtakes only part of the leading edge of the signal pulse. In the white region on the other hand, complete overtaking takes place, however, increasingly closer to the waveguide output for increasing $\Delta \tau$. Because of the nonlinear depletion of the switch, the induced index change $\Delta n_{\mathrm{FC}}$ and the resulting separation between the bands of Fig. 2(a) decrease towards the waveguide output, leading to a decrease of $\left|\Delta \lambda_{c}\right|$ with increasing $\Delta \tau$. We observe a maximum of $\left|\Delta \lambda_{c}\right|=0.9 \mathrm{~nm}$ at $\Delta \tau_{0}=1.4 \mathrm{ps}$. The corresponding spectrum, plotted in Fig. 2(g), is clearly blueshifted with respect to the spectra at $\Delta \tau_{\max }$ and $\Delta \tau_{\min }$, and the low peak transmission is, again, due to free carrier induced losses.

We estimate the maximum index change induced by the switch by considering an effective mode area of $0.25 \mu \mathrm{m}^{2}$ and a two-photon absorption coefficient of $0.7 \mathrm{~cm} / \mathrm{GW}$ [25], and obtain $\Delta n_{\mathrm{FC}, \max } \approx-3 \times 10^{-3}$. Further, we calculate the band diagrams for the designed photonic crystal waveguide and graphically calculate an expected wavelength shift of $-0.8 \mathrm{~nm}$, which matches well the value of $-0.9 \mathrm{~nm}$ we measured in Fig. 2(b).

Next, we implement the configurations of Figs. 2(c)

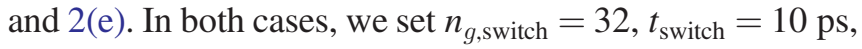
$P_{\text {switch }}=3.75 \mathrm{~W}$, and $t_{\text {signal }} \leq 7 \mathrm{ps}$. Additionally, taking into account an effective mode area of the switch of $0.50 \mu \mathrm{m}^{2}$, we estimate the same index change $\Delta n_{\mathrm{FC}, \max } \approx-3 \times 10^{-3}$ as in the case of Figs. 2(a), 2(b), and $2(\mathrm{~g})$. We choose different signal group indices in both cases, for the case of Fig. 2(c), $n_{g \text {,signal }}=11$ and
$\Delta \tau_{\mathrm{WG}}\left(n_{g, \text { signal }}=11\right)=28 \mathrm{ps}$, and in the case of Fig. 2(e), $n_{g, \text { signal }}=26$ and $\Delta \tau_{\mathrm{WG}}\left(n_{g, \text { signal }}=26\right)=10$ ps.

The measured wavelength shift $\Delta \lambda_{c}(\Delta \tau)$ is shown in Figs. 2(d) and 2(f) for $n_{g \text {,signal }}=11$ and $n_{g \text {,signal }}=26$, respectively. Again, overtaking takes place in the white regions, and the largest observed shift is $\Delta \lambda_{c}=-0.48 \mathrm{~nm}$ at $\Delta \tau_{0}=-0.5 \mathrm{ps}$ for Fig. 2(d) and $\Delta \lambda_{c}=-0.94 \mathrm{~nm}$ at $\Delta \tau_{0}=0$ ps for Fig. 2(f). In Figs. 2(h) and 2(i) we plot the signal spectra measured at $\Delta \tau_{\max }, \Delta \tau_{0}$, and $\Delta \tau_{\min }$ for $n_{g, \text { signal }}=11$ and $n_{g, \text { signal }}=26$, respectively.

The calculated expected shift of the signal wavelength is $-0.55 \mathrm{~nm}$ for $n_{g \text {,signal }}=11$, which matches well the measured $\Delta \lambda_{c}=-0.48 \mathrm{~nm}$. The shift of wave vector expected in this case is $0.05 \mu \mathrm{m}^{-1}$, and corresponds to the value we reported above. For $n_{g \text {,signal }}=26$, the largest measured $\left|\Delta \lambda_{c}\right|=0.94 \mathrm{~nm}$ is smaller than the expected $\left|\Delta \lambda_{c}\right| \sim 5.5 \mathrm{~nm}$, however. Correspondingly, the achieved shift of wave vector is smaller than the expected value of $0.5 \mu \mathrm{m}^{-1}$. The reason for this is that different parts of the signal pulse overtake the moving front at different positions of the waveguide, and, due to attenuation of the switching pulse, thus see a decreasing index contrast of the front $\Delta n_{\mathrm{FC}}$. However, for the same $P_{\text {switch }}$ and thus $\Delta n_{\mathrm{FC}}$, it is remarkable that the maximum $\left|\Delta \lambda_{c}\right|$ is larger for $n_{g, \text { signal }}=$ 26 than for $n_{g \text {,signal }}=11$, as predicted by theory. Finally, we emphasize that the observation of a blueshift in Figs. 2(d), 2(f), 2(h), and 2(i) unequivocally demonstrates that an indirect transition has actually been achieved.

The limitation imposed by the attenuation of the switching pulse during propagation through the waveguide, as observed in Figs. 2(e), 2(f), and 2(i), could be overcome by pumping the structure from the top with a pulse propagating along a direction tilted to the normal of the plane in which the waveguide resides [10]. Here, the propagation velocity of the front could be controlled by varying the tilt angle. With this modification of the experimental setup, the shifts of $5.5 \mathrm{~nm}(\sim 700 \mathrm{GHz})$ in wavelength and $0.5 \mu \mathrm{m}^{-1}$ in wave vector theoretically expected for the case of Fig. 2(e) can be achieved in a $396 \mu \mathrm{m}$ long structure by applying $\Delta n=-3 \times 10^{-3}$, as mentioned in the beginning.

In conclusion, we have experimentally achieved indirect photonic transitions that are driven by an optically generated refractive index front moving along a photonic crystal waveguide, and have demonstrated that the induced transitions depend on the propagation velocity of the front and on the photonic bands of the structure. Finally, we would like to emphasize some applications of fundamental physical and technological relevance which are opened up by this work. By drawing a phase continuity line with a slope of $-v_{f}$ in Fig. 2(a), it can be seen that different indirect photonic transitions are achieved for co- and counterpropagating light signal and refractive index front. In combination with a spectral filter, our device is therefore interesting for implementing optical isolation without using magneto-optical effects. Furthermore, slow light 
waveguides allow for tailoring of photonic bands with regions of different group velocities, such that an optical delay can be realized by inducing indirect transitions between these regions [17]. Varying the front velocity additionally allows for tuning of the delay, and even for stopping light by inducing an indirect transition to a region of zero group velocity. In multimode structures, the phase continuity line intersects several dispersion bands, and might even lead to an inversely Doppler shifted frequency $[21,26]$. In addition, by choosing $\left(\omega_{1}, k_{1}\right)$ in Fig. 2(a) to lie closer to the knee of the solid band, an indirect transition to the same photonic band can be achieved without crossing the dashed band, similar to [27]. Thus, a large frequency shift could be achieved with a small $\Delta n$. Finally, the reflection from a moving front that opens up a band gap in the medium where the light propagates allows for significantly manipulating the bandwidth of an optical signal while preserving its center frequency [28], a key feature that is of interest, for instance, in optical communication.

M. C. M., A. Y. P., and M. E. acknowledge the support of the German Research Foundation under Grant No. EI 391/ 13-2, and appreciate the support of CST, Darmstadt, Germany, with their Finite Integration Software Microwave Studio. J. L. acknowledges the support of the National Key Basic Research Special Foundation (2010CB923200), the National Natural Science Foundation of China (11204386), and Guangdong Natural Science Foundation (S2012040007812). T.F. K. and L. O'F acknowledge support by the EPSRC through the U.K. Silicon Photonics consortium.

*castellanos@tu-harburg.de

${ }^{\dagger}$ lijt3@mail.sysu.edu.cn

[1] J. N. Winn, S. Fan, J. D. Joannopoulos, and E. P. Ippen, Phys. Rev. B 59, 1551 (1999).

[2] Z. Yu and S. Fan, Nat. Photonics 3, 91 (2009).

[3] Z. Yu and S. Fan, IEEE J. Sel. Top. Quantum Electron. 16, 459 (2010).

[4] H. Lira, Z. Yu, S. Fan, and M. Lipson, Phys. Rev. Lett. 109, 033901 (2012).

[5] D. M. Beggs, I. H. Rey, T. Kampfrath, N. Rotenberg, L. Kuipers, and T. F. Krauss, Phys. Rev. Lett. 108, 213901 (2012).
[6] R. Stolte and R. Ulrich, Electron. Lett. 33, 1217 (1997).

[7] M. Lampe, E. Ott, and J. H. Walker, Phys. Fluids 21, 42 (1978).

[8] D. Hashimshony, A. Zigler, and K. Papadopoulos, Phys. Rev. Lett. 86, 2806 (2001).

[9] I. Geltner, Y. Avitzour, and S. Suckewer, Appl. Phys. Lett. 81, 226 (2002).

[10] J. Bae, Y. J. Xian, S. Yamada, and R. Ishikawa, Appl. Phys. Lett. 94, 091120 (2009).

[11] R. Soref and B. Bennett, IEEE J. Quantum Electron. 23, 123 (1987).

[12] M. Notomi and S. Mitsugi, Phys. Rev. A 73, 051803 (2006).

[13] S. F. Preble, Q. Xu, and M. Lipson, Nat. Photonics 1, 293 (2007).

[14] M. Castellanos Muñoz, A. Y. Petrov, and M. Eich, Appl. Phys. Lett. 101, 141119 (2012).

[15] J. Upham, Y. Tanaka, T. Asano, and S. Noda, Appl. Phys. Express 3, 062001 (2010).

[16] T. Kampfrath, D. M. Beggs, T. P. White, A. Melloni, T. F. Krauss, and L. Kuipers, Phys. Rev. A 81, 043837 (2010).

[17] K. Kondo, M. Shinkawa, Y. Hamachi, Y. Saito, Y. Arita, and T. Baba, Phys. Rev. Lett. 110, 053902 (2013).

[18] A. Y. Petrov and M. Eich, Appl. Phys. Lett. 85, 4866 (2004).

[19] J. Li, T. P. White, L. O'Faolain, A. Gomez-Iglesias, and T. F. Krauss, Opt. Express 16, 6227 (2008).

[20] W. B. Mori, T. Katsouleas, J. M. Dawson, and C. H. Lai, Phys. Rev. Lett. 74, 542 (1995).

[21] N. Seddon and T. Bearpark, Science 302, 1537 (2003).

[22] See Supplemental Material at http://link.aps.org/ supplemental/10.1103/PhysRevLett.112.053904 for a description of the waveguide geometry and of the experimental setup.

[23] L. O'Faolain et al., Opt. Express 18, 27627 (2010).

[24] A. Gomez-Iglesias, D. O’Brien, L. O'Faolain, A. Miller, and T. F. Krauss, Appl. Phys. Lett. 90, 261107 (2007).

[25] A. C. Turner-Foster, M. A. Foster, J. S. Levy, C. B. Poitras, R. Salem, A. L. Gaeta, and M. Lipson, Opt. Express 18, 3582 (2010).

[26] E. J. Reed, M. Soljacic, and J. D. Joannopoulos, Phys. Rev. Lett. 91, 133901 (2003).

[27] T. G. Philbin, C. Kuklewicz, S. Robertson, S. Hill, F. König, and U. Leonhardt, Science 319, 1367 (2008).

[28] E. A. Ulchenko, A. Yu. Petrov, M. Castellanos Muñoz, S. Lang, and M. Eich (unpublished). 